\title{
CONTRACTIVE METRICS FOR A BOLTZMANN EQUATION FOR GRANULAR GASES: DIFFUSIVE EQUILIBRIA.
}

\author{
M. BISI, J. A. CARRILLO AND G. TOSCANI
}

\begin{abstract}
We quantify the long-time behavior of a system of (partially) inelastic particles in a stochastic thermostat by means of the contractivity of a suitable metric in the set of probability measures. Existence, uniqueness, boundedness of moments and regularity of a steady state are derived from this basic property. The solutions of the kinetic model are proved to converge exponentially as $t \rightarrow \infty$ to this diffusive equilibrium in this distance metrizing the weak convergence of measures. Then, we prove a uniform bound in time on Sobolev norms of the solution, provided the initial data has a finite norm in the corresponding Sobolev space. These results are then combined, using interpolation inequalities, to obtain exponential convergence to the diffusive equilibrium in the strong $L^{1}$-norm, as well as various Sobolev norms.
\end{abstract}

\section{Introduction}

This paper concerns the long-time asymptotics of the the homogeneous Boltzmann equation for inelastic interactions in the pseudo-Maxwellian approximation introduced in [4]. Without an energy source into the system, the inelasticity of the collisions will homogenize the velocity of particles and thus, the only possible steady state is the Delta Dirac distribution at the mean velocity of the particles [4]. Therefore, we assume that our particles are under the influence of an external source of energy, a thermostat, that keeps the motion of particles. A stochastic thermostat was indeed proposed in different granular gases models as a candidate for keeping the temperature of particles bounded away from zero [1, 2, 22]. From a probabilistic point of view, a stochastic thermostat means that particles follow Brownian paths between collisions.

At the kinetic level the thermostat is represented by a linear diffusion term in velocity [11], that may be temperature dependent [20]. Hence, if $f(v, t) \geq 0$ denotes the density for the velocity space distribution of the molecules of the granular gas at time $t$, the time evolution of $f$ obeys the 
equation

$$
\frac{\partial f}{\partial t}=B \sqrt{\theta(t)} Q(f, f)+F \theta^{p}(t) \triangle_{v} f \quad \text { with } \quad 0 \leq p<\frac{3}{2},
$$

where $Q(f, f)$, the Boltzmann collision operator in the pseudo-Maxwellian approximation, models the effects of inelastic binary collisions between particles. We keep the positive constants $B$ and $F$, measuring the importance ratio of inelastic collisions and stochastic thermostat to keep track of their influences in the convergence rates. Instead of writing explicitly the collision operator [4], it is much easier (and less painful) to work in the corresponding weak form of the $Q(f, f)$. Thus, we will define $Q(f, f)$ by its weak form given by

$$
\langle\varphi, Q(f, f)\rangle=\frac{1}{4 \pi} \int_{\mathbb{R}^{3}} \int_{\mathbb{R}^{3}} \int_{S^{2}} f(v) f(w)\left[\varphi\left(v^{\prime}\right)-\varphi(v)\right] d n d v d w
$$

acting on functions $\varphi \in C\left(\mathbb{R}^{3}\right)$ where

$$
v^{\prime}=\frac{1}{2}(v+w)+\frac{1-e}{4}(v-w)+\frac{1+e}{4}|v-w| n
$$

is the postcollisional velocity and $0<e<1$ is the constant restitution coefficient. The unit vector $n$ in (1.3) parameterizes the set of all kinematically possible (i.e. those conserving mass and momentum) post-collisional velocities. Without loss of generality, in what follows we will assume that the initial datum $f_{0}$ is a probability density. By mass conservation, $f(v, t)$ will remain a probability density at any subsequent time.

The existence of steady states for equation (1.1) has been addressed by fixed point arguments in [14] and by moment methods in [5]. Uniqueness of stationary solutions was pointed out as an open problem in [14] and later addressed indirectly in [5] as a consequence of the uniqueness of the moment expansion for solutions of the initial value problem to (1.1). Recently, the existence of homogenous cooling states (HCS) (self-similar solutions) without the diffusion term was established in [6] and subsequently, proved in [7] that they give the intermediate asymptotic of the solutions, that is, the difference between any solution and the HCS measured in a suitable way vanishes as $t \rightarrow \infty$. Let us finally mention that the mathematical properties of the IVP for the more realistic hard-spheres model with diffusion have been studied recently in [16] and its tail behavior well described both in the stationary and the time-dependent case $[8,16,17]$. However, the long-time asymptotic in this case are not well understood since even the uniqueness of steady solutions for a given mean velocity remains an open problem.

In the rest of the paper, instead of working on the weak form (1.2), we will equivalently use the Fourier transformed equation corresponding 
to $(1.1)[3,4]$, that reads

$$
\frac{\partial \hat{f}}{\partial t}=B \sqrt{\theta(t)} \frac{1}{4 \pi} \int_{S^{2}}\left\{\hat{f}\left(t, k_{-}\right) \hat{f}\left(t, k_{+}\right)-\hat{f}(t, 0) \hat{f}(t, k)\right\} d n-F \theta^{p}(t)|k|^{2} \hat{f}
$$

with

$$
\begin{aligned}
& k_{-}=\frac{1+e}{4}(k-|k| n), \\
& k_{+}=\frac{3-e}{4} k+\frac{1+e}{4}|k| n .
\end{aligned}
$$

Our convention with the Fourier transform is that

$$
\hat{f}(k, t)=\int_{\mathbb{R}^{3}} f(v, t) e^{-i v \cdot k} d v
$$

This weak form of equation (1.1) is suitable to show both the existence and uniqueness of a steady state and its regularity. A crucial role in our analysis is played by the weak norm convergence, which is obtained by adapting a method first used in [15] to control the exponential convergence for Maxwellian molecules in certain weak norms. The global asymptotical stability of the unique steady state can be recovered in terms of the contractivity properties of these distances in Fourier space. From their introduction on, these distances were subsequently used for the classical Boltzmann equation for Maxwellian molecules in [9, 21], for the Fokker-Planck equation in [12] and for the Kac model for inelastic interactions recently in [19]. Contractivity of equivalent metrics for solutions of a general family of PDE models, including simplified models of granular media, has recently been shown in $[10,18,13]$ to produce results which are reminiscent of the main theorem in this work.

Let $\mathcal{P}_{s}\left(\mathbb{R}^{3}\right)$ be the set of probability measures with bounded $s$-moment. The Fourier-based metrics $d_{s}$, for any $s>0$, are defined as

$$
d_{s}(\hat{f}, \hat{g})=\sup _{k \in \mathbb{R}^{3}} \frac{|\hat{f}(k)-\hat{g}(k)|}{|k|^{s}}
$$

for any pair of probability measures in $\mathcal{P}_{s}\left(\mathbb{R}^{3}\right)$. This distance is well-defined and finite by simple Taylor expansion for any pair of probability measures with equal moments up to order $[s]$, where $[s]$ denotes the integer part of $s$. Moreover, in case $s \geq 1$ be an integer, it suffices equality of moments up to order $s-1$ for being $d_{s}$ finite.

In fact, $d_{2}$ topology is equivalent to the weak-star topology for measures plus boundedness of second moments [21], and can be related to the well-known Wasserstein distance between probability measures. The main results of this work can be summarized as follows: 
Theorem 1.1. Equation (1.1) has a unique steady state $f_{\infty}$ in the set of probability measures with a given mean velocity $u$. Moreover, $f_{\infty}$ is smooth and fast decaying at $\infty$. Furthermore, given any probability measure solution $f(t, v)$ of (1.1) with mean velocity $u$ and finite temperature, $d_{2}\left(\hat{f}(t), \hat{f}_{\infty}\right)$ tends exponentially to 0 . The rate $\lambda$ of exponential convergence is explicitly computable.

The key idea to prove this theorem is based on the observation that the $d_{2}$ distance is a contraction for the flow map of (1.1) restricted to initial data with equal temperature. This property will enable us to show the existence and uniqueness of the steady states by simple arguments invoking Banach fixed point theorem. The first step in our analysis will be the study of the time evolution of moments. We recover briefly the exact general moment equation of second order, and prove that the isotropic moments of any order satisfy a differential inequality which implies a uniform bound in time. These arguments will be described in Section 2. Section 3 deals with the existence of moments and regularity of the steady state. These properties are addressed directly on the integral equation in Fourier representation verified by the steady state itself. Section 4 is devoted to prove the exponential convergence towards steady state for any solution of (1.1). This is achieved by a careful estimate of the convergence of the temperature to the asymptotic steady value and using a variation of the argument proving the contractivity of the $d_{2}$ distance. Section 5 deals with the problem of obtaining strong convergence towards the steady state. This goal will be reached by establishing a propagation of smoothness result for (1.1). To state the result concisely, we introduce the Sobolev space norms $\|\cdot\|_{H^{r}\left(\mathbb{R}^{3}\right)}$, $r \geq 0$ by

$$
\|f\|_{H^{r}\left(\mathbb{R}^{3}\right)}=\int_{\mathbb{R}^{3}}|k|^{2 r}|\hat{f}(k)|^{2} d k,
$$

then we prove

Theorem 1.2. Let $f_{0}$ be any initial datum for equation (1.1) with $\left\|f_{0}\right\|_{H^{r}\left(\mathbb{R}^{3}\right)}$ finite. Then, any probability density solution $f(t, v)$ of $(1.1)$ is bounded in $H^{r}$, and there is a universal constant $C_{r}$ so that, for all $t>0$,

$$
\|f(t)\|_{H^{r}\left(\mathbb{R}^{3}\right)} \leq \max \left\{\left\|f_{0}\right\|_{H^{r}\left(\mathbb{R}^{3}\right)}, C_{r}\right\} .
$$

The results of Theorems 1.1 and 1.2 will then be combined, using interpolation inequalities [9], to obtain exponential convergence to $f_{\infty}$ in the strong $L^{1}$-norm, as well as in various Sobolev norms. 
Theorem 1.3. Let $f_{0}$ be any initial datum for equation (1.1). Let $\epsilon>0$ be given. Then, there is a number $r$ depending only on $\epsilon$ so that whenever

$$
\int_{\mathbb{R}^{3}}|v|^{2 r} f_{0}(v) d v+\int_{\mathbb{R}^{3}}|k|^{2 r}\left|\hat{f}_{0}(k)\right|^{2} d k<\infty
$$

then it holds that

$$
\left\|f(\cdot, t)-f_{\infty}\right\|_{L^{1}\left(\mathbb{R}^{3}\right)} \leq C_{\epsilon} \exp \{-(1-\epsilon) \lambda t\} .
$$

Here, $\lambda$ is the rate of exponential convergence in $d_{2}$ distance, and $C_{\epsilon}$ is explicitly computable in terms of the integrals specified above. Moreover, increasing $r$, we obtain the same result if the $L^{1}$-norm is replaced by any $H^{m}$-norm.

\section{Evolution OF MOMENTS}

Let us denote by $\rho(t), u(t), \theta(t)$ and $p_{i j}(t)$ the density, mean velocity, temperature and pressure tensor components of the distribution function $f$. Since mass and mean velocity are preserved clearly by the collision operator (1.2) and we deal with the homogeneous problem, without loss of generality we assume in the rest of the paper that $\rho(t)=1$ and $u(t)=0$ for all $t \geq 0$. Following [5], we compute the general moment equations of second order.

Lemma 2.1. [Evolution of moments [11, 5]] Let $\hat{f}$ be a solution to (1.4) with unit mass, zero mean velocity and initial second order moments bounded, then $\hat{f}$ has finite second order moments for any $t>0$,

$$
\frac{d \theta}{d t}=-\frac{1-e^{2}}{4} B \theta^{\frac{3}{2}}+2 F \theta^{p},
$$

and

$$
\begin{aligned}
\frac{\partial}{\partial t} \int_{\mathbb{R}^{3}} f(v) v_{i} v_{j} d v=- & \frac{(1+e)(3-e)}{8} B \sqrt{\theta_{f}(t)} \int_{\mathbb{R}^{3}} f(v) v_{i} v_{j} d v \\
& +\delta_{i j} 2\left(\frac{1+e}{4}\right)^{2} B\left(\theta_{f}(t)\right)^{\frac{3}{2}}+\delta_{i j} 2 F \theta_{f}^{p}(t)
\end{aligned}
$$

for any $t \geq 0$. As a consequence, we have the following asymptotically stable steady value of the temperature

$$
\theta_{\infty}=\left(\frac{8 F}{B\left(1-e^{2}\right)}\right)^{\frac{2}{3-2 p}}
$$


Proof.- By multiplying (1.1) by $v_{i} v_{j}$ and integrating we get

$$
\begin{array}{r}
\frac{\partial}{\partial t} \int_{\mathbb{R}^{3}} f(v) v_{i} v_{j} d v=\frac{B}{4 \pi} \sqrt{\theta_{f}(t)} \int_{\mathbb{R}^{3}} \int_{\mathbb{R}^{3}} \int_{S^{2}} f(v) f(w)\left[v_{i}^{\prime} v_{j}^{\prime}-v_{i} v_{j}\right] d n d v d w \\
+F \theta_{f}^{p}(t) \int_{\mathbb{R}^{3}} v_{i} v_{j} \triangle_{v} f d v .
\end{array}
$$

Since

$$
v^{\prime}=\frac{3-e}{4} v+\frac{1+e}{4} w+\frac{1+e}{4}|v-w| n
$$

we have

$$
\begin{aligned}
\frac{\partial}{\partial t} \int_{\mathbb{R}^{3}} f(v) v_{i} v_{j} d v & \\
= & \frac{B}{4 \pi} \sqrt{\theta_{f}(t)} \int_{\mathbb{R}^{3}} \int_{\mathbb{R}^{3}} \int_{S^{2}} f(v) f(w)\left\{\left[\left(\frac{3-e}{4}\right)^{2}-1\right] v_{i} v_{j}\right. \\
& +\left(\frac{1+e}{4}\right)^{2}\left(w_{i} w_{j}+|v-w|^{2} n_{i} n_{j}+|v-w|\left(w_{i} n_{j}+w_{j} n_{i}\right)\right) \\
& \left.+\left(\frac{(3-e)(1+e)}{16}\right)\left(v_{i} w_{j}+w_{i} v_{j}+|v-w|\left(v_{i} n_{j}+v_{j} n_{i}\right)\right)\right\} d n d v d w \\
& +\delta_{i j} 2 F \theta_{f}^{p}(t) .
\end{aligned}
$$

Evolution equations (2.1) and (2.2) are then obtained imposing zero mean velocity and unit mass, while boundedness of second moments is an easy consequence of (2.1).

Remark 2.2. [Temperature for the case $\boldsymbol{p}=1$ ] If $p=1$, the evolution equation (2.1) for the temperature can be solved in analytic way. In fact, if we put $z=\theta \mathrm{e}^{-2 F t}$, we have

$$
\frac{d z}{d t}=-\frac{1-e^{2}}{4} B \mathrm{e}^{F t} z^{\frac{3}{2}}
$$

from which with trivial calculations it follows:

$$
z(t)=\left[z_{0}^{-\frac{1}{2}}+\frac{1-e^{2}}{8} \frac{B}{F}\left(\mathrm{e}^{F t}-1\right)\right]^{-2}
$$


namely the analytic expression of the temperature is

$$
\theta(t)=\left[\frac{\mathrm{e}^{F t}}{\theta_{0}^{-\frac{1}{2}}+\frac{1-e^{2}}{8} \frac{B}{F}\left(\mathrm{e}^{F t}-1\right)}\right]^{2} .
$$

For future reference we include here the computation:

$$
\int_{0}^{t} \sqrt{\theta(\tau)} d \tau=\frac{8}{\left(1-e^{2}\right) B} \log \left[1+\frac{1-e^{2}}{8 \theta_{0}^{-\frac{1}{2}}} \frac{B}{F}\left(\mathrm{e}^{F t}-1\right)\right]
$$

The exact evolution equations for moments of order higher than two has been done in [5] only for the third order. Evolution of higher order moments has been studied in [5] only in the relatively simple case of isotropic solutions. We obtain here an explicit inequality for the time evolution of isotropic moments of any order for general solutions, which leads to prove a uniform bound in time of these moments, in terms of the moments of the initial value. To simplify notations, in what follows we denote

$$
M_{2 r}(t)=\int_{\mathbb{R}^{3}} f(v, t)|v|^{2 r} d v
$$

for any $r \in \mathbb{N}$. Moreover, we will take advantage of the weak form of the collision integral

$$
\langle\varphi, Q(f, f)\rangle=\frac{1}{8 \pi} \int_{\mathbb{R}^{3}} \int_{\mathbb{R}^{3}} \int_{S^{2}} f(v) f(w)\left[\varphi\left(v^{\prime}\right)+\varphi\left(w^{\prime}\right)-\varphi(v)-\varphi(w)\right] d n d v d w
$$

acting on functions $\varphi \in C\left(\mathbb{R}^{3}\right)$. Here $\left(v^{\prime}, w^{\prime}\right)$ represent the post-collisional velocities, $v^{\prime}$ is given by $(1.3)$, and

$$
w^{\prime}=\frac{1}{2}(v+w)-\frac{1-e}{4}(v-w)-\frac{1+e}{4}|v-w| n
$$

The weak form (2.6) has been considered in [4], and is equivalent to (1.2). We prove

Lemma 2.3. [Uniform in time moment estimates] Let $f(t, v)$ be the solution to equation (1.1), where the initial distribution $f_{0}(v)$ is such that

$$
\int_{\mathbb{R}^{3}} f_{0}(v)|v|^{2 r} d v=c_{r}<+\infty
$$


for some $r \geq 2$. Then, $M_{2 r}(t)$ satisfies the following differential inequality

$$
\begin{aligned}
\frac{d}{d t} M_{2 r}(t) \leq & -B \sqrt{\frac{M_{2}(t)}{3}}\left[\frac{1-e^{2 r}}{4}\left(M_{2 r}(t)+M_{2(r-1)}(t) M_{2}(t)\right)\right. \\
& \left.-\frac{1}{2} \sum_{m=1}^{r-1}\left(\begin{array}{c}
r \\
m
\end{array}\right) M_{2(r-m)}(t) M_{2 m}(t)\right] \\
& +F M_{2}(t)^{p}\left(2 r+4 r^{2}\right) M_{2(r-1)}(t) .
\end{aligned}
$$

Consequently, $M_{2 r}(t)$ is uniformly bounded in time.

Proof.- Elementary computations show that

$$
0 \geq\left(v^{\prime}\right)^{2}+\left(w^{\prime}\right)^{2}-\left(v^{2}+w^{2}\right)=-\frac{1-e^{2}}{4}\left[(v-w)^{2}-|v-w|(v-w) \cdot n\right],
$$

and

$$
\left(v^{\prime}\right)^{2}+\left(w^{\prime}\right)^{2} \geq e^{2}\left(v^{2}+w^{2}\right) .
$$

Inequality (2.11) follows from (2.10). In fact, since

$$
0 \leq\left[(v-w)^{2}-|v-w|(v-w) \cdot n\right] \leq 2(v-w)^{2},
$$

we obtain

$$
\begin{aligned}
\left(v^{\prime}\right)^{2}+\left(w^{\prime}\right)^{2} & \geq\left(v^{2}+w^{2}\right)-\frac{1-e^{2}}{2}(v-w)^{2}=\frac{1}{2}(v+w)^{2}+\frac{e^{2}}{2}(v-w)^{2} \\
& \geq e^{2}\left(v^{2}+w^{2}\right)
\end{aligned}
$$


Choosing $\varphi(v)=|v|^{2 r}, r \geq 2$, we obtain

$$
\begin{aligned}
\left\langle|v|^{2 r}, Q\right. & (f, f)\rangle= \\
= & \frac{1}{8 \pi} \int_{\mathbb{R}^{3}} \int_{\mathbb{R}^{3}} \int_{S^{2}} f(v) f(w)\left[\left|v^{\prime}\right|^{2 r}+\left|w^{\prime}\right|^{2 r}-|v|^{2 r}+|w|^{2 r}\right] d n d v d w \\
= & \frac{1}{8 \pi} \int_{\mathbb{R}^{3}} \int_{\mathbb{R}^{3}} \int_{S^{2}} f(v) f(w)\left[\left(\left|v^{\prime}\right|^{2}+\left|w^{\prime}\right|^{2}\right)^{r}-\left(|v|^{2}+|w|^{2}\right)^{r}\right] d n d v d w \\
& +\sum_{m=1}^{r-1}\left(\begin{array}{c}
r \\
m
\end{array}\right) \frac{1}{8 \pi} \int_{\mathbb{R}^{3}} \int_{\mathbb{R}^{3}} \int_{S^{2}} f(v) f(w)\left[|v|^{2(r-m)}|w|^{2 m}\right. \\
\leq & \frac{1}{8 \pi} \int_{\mathbb{R}^{3}} \int_{\mathbb{R}^{3}} \int_{S^{2}} f(v) f(w)\left[\left(\left|v^{\prime}\right|^{2(r-m)}+\left|w^{\prime}\right|^{2 m}\right] d n d v d w\right. \\
& \left.+\frac{1}{2} \sum_{m=1}^{r-1}\left(\begin{array}{c}
r \\
m
\end{array}\right) M_{2(r-m)} M_{2 m} M^{r}-\left(|v|^{2}+|w|^{2}\right)^{r}\right] d n d v d w
\end{aligned}
$$

Taking into account (2.11), we obtain

$$
\begin{aligned}
& \frac{1}{8 \pi} \int_{S^{2}}\left[\left(\left|v^{\prime}\right|^{2}+\left|w^{\prime}\right|^{2}\right)^{r}-\left(|v|^{2}+|w|^{2}\right)^{r}\right] d n \\
& =\frac{1}{8 \pi} \int_{S^{2}}\left[\left|v^{\prime}\right|^{2}+\left|w^{\prime}\right|^{2}-\left(|v|^{2}+|w|^{2}\right)\right] \sum_{m=0}^{r-1}\left[\left|v^{\prime}\right|^{2}+\left|w^{\prime}\right|^{2}\right]^{r-m-1}\left[|v|^{2}+|w|^{2}\right]^{m} d n \\
& \leq \frac{1}{8 \pi} \int_{S^{2}}\left[\left|v^{\prime}\right|^{2}+\left|w^{\prime}\right|^{2}-\left(|v|^{2}+|w|^{2}\right)\right] \sum_{m=0}^{r-1} e^{2(r-m-1)}\left[|v|^{2}+|w|^{2}\right]^{r-1} d n \\
& =-\frac{1-e^{2 r}}{4}\left[|v|^{2}+|w|^{2}\right]^{r-1} \frac{1}{8 \pi} \int_{S^{2}}\left[(v-w)^{2}-|v-w|(v-w) \cdot n\right] d n \\
& =-\frac{1-e^{2 r}}{8}\left[|v|^{2}+|w|^{2}\right]^{r-1}(v-w)^{2} .
\end{aligned}
$$


Thus, inserting (2.13) into (2.12) we obtain the inequality

$$
\begin{array}{r}
\left\langle|v|^{2 r}, Q(f, f)\right\rangle \leq-\frac{1-e^{2 r}}{8} \int_{\mathbb{R}^{3}} \int_{\mathbb{R}^{3}} f(v) f(w)\left[|v|^{2}+|w|^{2}\right]^{r-1}(v-w)^{2} d v d w \\
+\frac{1}{2} \sum_{m=1}^{r-1}\left(\begin{array}{c}
r \\
m
\end{array}\right) M_{2(r-m)} M_{2 m} .
\end{array}
$$

Finally, since

$$
\begin{gathered}
\int_{\mathbb{R}^{3}} \int_{\mathbb{R}^{3}} f(v) f(w)\left[|v|^{2}+|w|^{2}\right]^{r-1}(v-w)^{2} d v d w \\
\geq \int_{\mathbb{R}^{3}} \int_{\mathbb{R}^{3}} f(v) f(w)\left[|v|^{2(r-1)}+|w|^{2(r-1)}\right](v-w)^{2} d v d w=2 M_{2 r}+2 M_{2(r-1)} M_{2} . \\
\left\langle|v|^{2 r}, Q(f, f)\right\rangle \leq-\frac{1-e^{2 r}}{4}\left(M_{2 r}+M_{2(r-1)} M_{2}\right)+\frac{1}{2} \sum_{m=1}^{r-1}\left(\begin{array}{c}
r \\
m
\end{array}\right) M_{2(r-m)} M_{2 m} .
\end{gathered}
$$

To conclude the proof, consider that

$$
\int_{\mathbb{R}^{3}}|v|^{2 r} \triangle_{v} f d v=\left(2 r+4 r^{2}\right) M_{2(r-1)} .
$$

Then, in order to show that $M_{2 r}(t)$ is uniformly bounded in time, one rewrites inequality $(2.9)$ as

$$
\frac{d}{d t} M_{2 r}(t) \leq A_{r}(t)\left[-M_{2 r}(t)+B_{r}(t)\right],
$$

where both $A_{r}(t)$ and $B_{r}(t)$ depend on moments up to the order $2(r-1)$. Thanks to Lemma 2.1, $M_{2}(t)$ is uniformly bounded in time. This implies that $B_{1}(t)$ is uniformly bounded in time, $B_{1}(t) \leq B_{1}$, and, consequently, $M_{4}(t)$ is uniformly bounded,

$$
M_{4}(t) \leq \max \left\{M_{4}(0), B_{1}\right\} .
$$

Recursively, the proof can be extended to any $r>2$. $\square$

\section{Non expansivity of the $\boldsymbol{d}_{2}$ Distance: Diffusive equilibria.}

The results of the previous section guarantee that the isotropic moments which are initially bounded, remain bounded in time. Here we concentrate on the time evolution of the $d_{2}$ distance. 
Proposition 3.1. [Uniform Contraction of the $\boldsymbol{d}_{2}$ distance] Let $\hat{f}_{1}$ and $\hat{f}_{2}$ be two solutions to (1.4) corresponding to initial values $\hat{f}_{1}(0), \hat{f}_{2}(0)$ with unit mass, zero mean velocity and equal temperature. Then $d_{2}\left(\hat{f}_{1}(0), \hat{f}_{2}(0)\right)<\infty$ and there exists a constant $C_{1}>0$ such that

$$
d_{2}\left(\hat{f}_{1}(t), \hat{f}_{2}(t)\right) \leq d_{2}\left(\hat{f}_{1}(0), \hat{f}_{2}(0)\right) \mathrm{e}^{-\left(1-\gamma_{0}\right) \mathrm{C}_{1} \mathrm{t}},
$$

for any $t \geq 0$. In (3.1), $\gamma_{0}=\left(e^{2}+3\right) / 4<1, C_{1}=B \min \left\{\theta_{0}, \theta_{\infty}\right\}^{1 / 2}$. In the particular case $p=1$ the more precise estimate holds

$$
d_{2}\left(\hat{f}_{1}(t), \hat{f}_{2}(t)\right) \leq d_{2}\left(\hat{f}_{1}(0), \hat{f}_{2}(0)\right)\left[1-\frac{1-e^{2}}{8 \theta_{0}^{-\frac{1}{2}}} \frac{B}{F}+\frac{1-e^{2}}{8 \theta_{0}^{-\frac{1}{2}}} \frac{B}{F} \mathrm{e}^{F t}\right]^{-2},
$$

for any $t \geq 0$.

Proof.- Since the evolution of the temperature is governed by the law given in Lemma 2.1 and the initial temperature of both solutions is equal, it follows that $\theta_{f}(t)=\theta_{g}(t)=\theta(t)$ for all $t \geq 0$. By the definition of the metric $d_{2}, d_{2}\left(\hat{f}_{1}(0), \hat{f}_{2}(0)\right)<\infty$ and we can subtract the equations satisfied by $\hat{f}_{1}(t)$ and $\hat{f}_{2}(t)$ respectively to get

$$
\begin{aligned}
\frac{\partial}{\partial t} \frac{\hat{f}_{1}(k)-\hat{f}_{2}(k)}{|k|^{2}}= & \frac{B}{4 \pi} \sqrt{\theta(t)} \int_{S^{2}}\left[\frac{\hat{f}_{1}\left(k_{-}\right) \hat{f}_{1}\left(k_{+}\right)-\hat{f}_{2}\left(k_{-}\right) \hat{f}_{2}\left(k_{+}\right)}{|k|^{2}}\right. \\
- & \left.\frac{\hat{f}_{1}(0) \hat{f}_{1}(k)-\hat{f}_{2}(0) \hat{f}_{2}(k)}{|k|^{2}}\right] d n \\
& -F \theta^{p}(t)|k|^{2} \frac{\hat{f}_{1}(k)-\hat{f}_{2}(k)}{|k|^{2}} .
\end{aligned}
$$

In the first term of the right-hand side, we do the usual splitting

$$
\begin{aligned}
& \left|\frac{\hat{f}_{1}\left(k_{-}\right) \hat{f}_{1}\left(k_{+}\right)-\hat{f}_{2}\left(k_{-}\right) \hat{f}_{2}\left(k_{+}\right)}{|k|^{2}}\right| \\
& \quad \leq\left|\hat{f}_{1}\left(k_{+}\right)\right| \frac{\left|\hat{f}_{1}\left(k_{-}\right)-\hat{f}_{2}\left(k_{-}\right)\right|}{\left|k_{-}\right|^{2}} \frac{\left|k_{-}\right|^{2}}{|k|^{2}}+\left|\hat{f}_{2}\left(k_{-}\right)\right| \frac{\left|\hat{f}_{1}\left(k_{+}\right)-\hat{f}_{2}\left(k_{+}\right)\right|}{\left|k_{+}\right|^{2}} \frac{\left|k_{+}\right|^{2}}{|k|^{2}} \\
& \quad \leq \sup _{k \in \mathbb{R}^{3}}\left\{\frac{\left|\hat{f}_{1}(k)-\hat{f}_{2}(k)\right|}{|k|^{2}}\left(\frac{\left|k_{-}\right|^{2}+\left|k_{+}\right|^{2}}{|k|^{2}}\right)\right\} .
\end{aligned}
$$


It is easy to check that

$$
\begin{aligned}
& \left|k_{-}\right|^{2}=|k|^{2}\left(\frac{1+e}{4}\right)^{2} 2(1-\cos \vartheta) \\
& \left|k_{+}\right|^{2}=|k|^{2}\left[\left(\frac{3-e}{4}\right)^{2}+\left(\frac{1+e}{4}\right)^{2}+2\left(\frac{3-e}{4}\right)\left(\frac{1+e}{4}\right) \cos \vartheta\right]
\end{aligned}
$$

where $\vartheta$ is the angle between the unit vectors $k /|k|$ and $n$. Therefore

$$
\frac{1}{4 \pi}\left|\int_{S^{2}} \frac{\hat{f}_{1}\left(k_{-}\right) \hat{f}_{1}\left(k_{+}\right)-\hat{f}_{2}\left(k_{-}\right) \hat{f}_{2}\left(k_{+}\right)}{|k|^{2}} d n\right| \leq \gamma_{0} \sup _{k \in \mathbb{R}^{3}} \frac{\left|\hat{f}_{1}(k)-\hat{f}_{2}(k)\right|}{|k|^{2}}
$$

where $\gamma_{0}$, for each restitution coefficient $e \neq 1$, denotes the constant

$$
\begin{aligned}
\gamma_{0}=\frac{1}{2} \int_{0}^{\pi}\left\{\left(\frac{1+e}{4}\right)^{2} 2(1-\cos \vartheta)\right. \\
\left.\quad+\left(\frac{3-e}{4}\right)^{2}+\left(\frac{1+e}{4}\right)^{2}+2\left(\frac{3-e}{4}\right)\left(\frac{1+e}{4}\right) \cos \vartheta\right\} \sin \vartheta d \vartheta \\
=\frac{1}{2} \int_{0}^{\pi}\left\{\frac{e^{2}+3}{4}+\frac{(1+e)(1-e)}{4} \cos \vartheta\right\} \sin \vartheta d \vartheta=\frac{e^{2}+3}{4}<1 .
\end{aligned}
$$

Let us set

$$
h(t, k)=\frac{\hat{f}_{1}(k)-\hat{f}_{2}(k)}{|k|^{2}} .
$$

Since $\hat{f}_{1}(0)=\hat{f}_{2}(0)=1$, equation $(3.3)$ becomes

$$
\left|\partial_{t} h(t, k)+\left(B \sqrt{\theta(t)}+F \theta^{p}(t)|k|^{2}\right) h(t, k)\right| \leq \gamma_{0} B \sqrt{\theta(t)}\|h(t, \cdot)\|_{\infty} .
$$

This is equivalent to

$$
\begin{aligned}
& \left|\partial_{t}\left[h(t, k) \exp \left(B \int_{0}^{t} \sqrt{\theta(s)} d s+F|k|^{2} \int_{0}^{t} \theta^{p}(s) d s\right)\right]\right| \\
& \quad \leq \gamma_{0} B \sqrt{\theta(t)}\|h(t, \cdot)\|_{\infty} \exp \left(B \int_{0}^{t} \sqrt{\theta(s)} d s+F|k|^{2} \int_{0}^{t} \theta^{p}(s) d s\right) .
\end{aligned}
$$


Integrating from 0 to $t$ we get

$$
\begin{gathered}
\left|h(t, k) \exp \left(B \int_{0}^{t} \sqrt{\theta(s)} d s+F|k|^{2} \int_{0}^{t} \theta^{p}(s) d s\right)\right| \leq|h(0, k)| \\
+\gamma_{0} B \int_{0}^{t} \sqrt{\theta(\tau)}\|h(\tau, \cdot)\|_{\infty} \exp \left(B \int_{0}^{\tau} \sqrt{\theta(s)} d s+F|k|^{2} \int_{0}^{\tau} \theta^{p}(s) d s\right) d \tau
\end{gathered}
$$

namely

$$
\begin{aligned}
& \exp \left(B \int_{0}^{t} \sqrt{\theta(s)} d s\right)|h(t, k)| \leq|h(0, k)| \\
& \quad+\gamma_{0} B \int_{0}^{t} \sqrt{\theta(\tau)}\|h(\tau, \cdot)\|_{\infty} \exp \left(B \int_{0}^{\tau} \sqrt{\theta(s)} d s\right) d \tau .
\end{aligned}
$$

Since the above inequality holds for all values of the variable $k$,

$$
\begin{aligned}
& \exp \left(B \int_{0}^{t} \sqrt{\theta(s)} d s\right)\|h(t, \cdot)\|_{\infty} \leq\|h(0, \cdot)\|_{\infty} \\
& +\gamma_{0} B \int_{0}^{t} \sqrt{\theta(\tau)}\|h(\tau, \cdot)\|_{\infty} \exp \left(B \int_{0}^{\tau} \sqrt{\theta(s)} d s\right) d \tau .
\end{aligned}
$$

Hence, if we put $w(t)=\exp \left(B \int_{0}^{t} \sqrt{\theta(s)} d s\right)\|h(t, \cdot)\|_{\infty}$

$$
w(t) \leq w(0)+\gamma_{0} B \int_{0}^{t} \sqrt{\theta(\tau)} w(\tau) d \tau .
$$

By the generalized Gronwall inequality, this implies

$$
w(t) \leq w(0) \exp \left(\gamma_{0} B \int_{0}^{t} \sqrt{\theta(\tau)} d \tau\right)
$$

namely

$$
\|h(t, \cdot)\|_{\infty} \leq\|h(0, \cdot)\|_{\infty} \exp \left(-\left(1-\gamma_{0}\right) B \int_{0}^{t} \sqrt{\theta(\tau)} d \tau\right) .
$$

Now, let us remark that the evolution equation (2.1) implies $\theta(t) \geq \tilde{\theta}$, where

$$
\tilde{\theta}=\min \left\{\theta_{0}, \theta_{\infty}\right\}
$$

and thus (3.1) is proved. In the particular case $p=1$ one can use the explicit expression of the integral in (2.5) to obtain (3.2). 
An important consequence of the uniform contraction of the $d_{2}$ distance through the flow of the Fourier version of the inelastic BE with thermal bath, is that we conclude both with the existence and uniqueness of the steady state (cf. $[5,14])$.

Let us denote by $X_{\theta}$ the subset of $\mathcal{P}_{2}\left(\mathbb{R}^{3}\right)$ given by all probability densities with zero mean velocity and temperature $\theta \in \mathbb{R}^{+}$. Let us consider

$$
H^{\infty}\left(\mathbb{R}^{3}\right)=\bigcap_{m=0}^{\infty} H^{m}\left(\mathbb{R}^{3}\right) .
$$

Theorem 3.2. [Existence, uniqueness and regularity of the steady state] Equation (1.1) has a unique steady state $f_{\infty}$ in the set of probability measures with zero mean velocity. Moreover, all the moments of $f_{\infty}$ are finite and $f_{\infty}$ is regular, i.e. $f_{\infty} \in H^{\infty}\left(\mathbb{R}^{3}\right)$.

Proof.- We split the proof into several steps.

Step 1: Existence and uniqueness of steady state. - Thanks to [21, Theorem 1], the set $X_{\theta_{\infty}}$ of $\mathcal{P}_{2}\left(\mathbb{R}^{3}\right)$ is closed with the distance $d_{2}$. Therefore, $\left(X_{\theta_{\infty}}, d_{2}\right)$ is a Banach metric space. Let us consider the flow map of (1.4),

$$
T_{t}:\left(X_{\theta_{\infty}}, d_{2}\right) \longrightarrow\left(X_{\theta_{\infty}}, d_{2}\right)
$$

for any time $t>0$, given by $T_{t}\left(f_{0}\right)=f(t)$ with $f(t)$ the unique solution at time $t$ of (1.4) with initial datum $f_{0} \in X_{\theta_{\infty}}$.

Proposition 3.1 proves that $T_{t}$ is a uniform contraction from the Banach space $\left(X_{\theta_{\infty}}, d_{2}\right)$ into itself with contraction constant $\mathrm{e}^{-\left(1-\gamma_{0}\right) \mathrm{Ct}}<1$. Therefore, Banach fixed point theorem ensures the existence and uniqueness of a unique steady state in $\left(X_{\theta_{\infty}}, d_{2}\right)$ and thus the first assertion of the theorem.

Step 2: Boundedness of all moments. - Thanks to Lemma 2.3, we know that the $m$-th isotropic moment remains uniformly bounded if the same moment is bounded initially. We can repeat the same argument of Proposition 3.1 , by computing now the evolution of the distance with index $2+m$ between any two solutions. One proves the following assertion: given any natural $m \geq 1$, let $\hat{f}_{1}(0)$ and $\hat{f}_{2}(0)$ be two initial data to $(1.4)$ with equal moments up to order $m+1$ and finite moments of order $m+2$, then $d_{2+m}\left(\hat{f}_{1}(0), \hat{f}_{2}(0)\right)<\infty$ and there exists a constant $C>0$ such that

$$
d_{2+m}\left(\hat{f}_{1}(t), \hat{f}_{2}(t)\right) \leq d_{2+m}\left(\hat{f}_{1}(0), \hat{f}_{2}(0)\right) \mathrm{e}^{-\left(1-\gamma_{m}\right) C t},
$$


for any $t \geq 0$, with

$$
\begin{aligned}
& \gamma_{m}=\frac{1}{2} \int_{0}^{\pi}\left\{\left[\left(\frac{1+e}{4}\right)^{2} 2(1-\cos \vartheta)\right]^{\frac{2+m}{2}}\right. \\
& \left.+\left[\left(\frac{3-e}{4}\right)^{2}+\left(\frac{1+e}{4}\right)^{2}+2\left(\frac{3-e}{4}\right)\left(\frac{1+e}{4}\right) \cos \vartheta\right]^{\frac{2+m}{2}}\right\} \sin \vartheta d \vartheta .
\end{aligned}
$$

Let us remark that the constant $C$ is the same for all $m$ and that $\gamma_{m}<$ $\gamma_{0}<1$ by definition. Let us also point out that (3.8) implies that moments of the solutions remain equal up to order $m+1, m \geq 1$, since initially they are equal, and thus, the distance $d_{2+m}\left(\hat{f}_{1}(t), \hat{f}_{2}(t)\right)<\infty$, for all $t \geq 0$.

Now, we proceed by induction. We already know that the steady state has temperature $\theta_{\infty}$. Let us assume that $f_{\infty}$ has moments bounded up to order $m+1$. Take the set $X_{\theta_{\infty}}^{m}$ defined as the subset of $\mathcal{P}_{2+m}\left(\mathbb{R}^{3}\right)$ with equal moments to those of $f_{\infty}$ up to order $m+1$. This set is a complete metric space endowed with the distance $d_{2+m}$ being a closed subset of $\mathcal{P}_{2+m}\left(\mathbb{R}^{3}\right)$. Proceeding as in the first step, the flow map $T_{t}$ is a uniform contraction from $\left(X_{\theta_{\infty}}^{m}, d_{2+m}\right)$ into itself with contraction constant less than 1 . Therefore, $T_{t}$ has a unique steady state $g \in X_{\theta}^{m}$ and thus, by uniqueness of steady state in $X_{\theta_{\infty}}$ we conclude $f_{\infty}=g \in\left(X_{\theta_{\infty}}^{m}, d_{2+m}\right)$ and thus, $f_{\infty}$ has finite moments of order $m+2$.

Step 3: Regularity. - In Fourier variables, the steady state $f_{\infty}$ satisfies the equation

$$
B \sqrt{\theta_{\infty}} \frac{1}{4 \pi} \int_{S^{2}}\left\{\hat{f}_{\infty}\left(k_{-}\right) \hat{f}_{\infty}\left(k_{+}\right)-\hat{f}_{\infty}(0) \hat{f}_{\infty}(k)\right\} d n-F \theta_{\infty}^{p}|k|^{2} \hat{f}_{\infty}(k)=0
$$

that, by virtue of $(2.3)$, simplifies to

$$
\int_{S^{2}} \hat{f}_{\infty}\left(k_{-}\right) \hat{f}_{\infty}\left(k_{+}\right) d n=4 \pi\left(1+\frac{1-e^{2}}{8} \theta_{\infty}|k|^{2}\right) \hat{f}_{\infty}(k) .
$$

Multiplying (3.11) by the conjugate of $\hat{f}_{\infty}$ (denoted by $\hat{f}_{\infty}^{c}$ ) and integrating over a ball $B_{R}$ of radius $R>0$ centered at zero, we get

$$
\int_{B_{R}} \int_{S^{2}} \hat{f}_{\infty}\left(k_{-}\right) \hat{f}_{\infty}\left(k_{+}\right) \hat{f}_{\infty}^{c}(k) d n d k=4 \pi \int_{B_{R}}\left(1+\frac{1-e^{2}}{8} \theta_{\infty}|k|^{2}\right)\left|\hat{f}_{\infty}(k)\right|^{2} d k
$$


Hence, the left-hand side is real, positive and finite for any $R>0$. On the other hand, we may directly estimate this left-hand side.

$$
\begin{aligned}
& \int_{B_{R}} \int_{S^{2}} \hat{f}_{\infty}\left(k_{-}\right) \hat{f}_{\infty}\left(k_{+}\right) \hat{f}_{\infty}^{c}(k) d n d k \\
\leq & \int_{B_{R}} \int_{S^{2}}\left|\hat{f}_{\infty}\left(k_{-}\right)\right|\left|\hat{f}_{\infty}\left(k_{+}\right)\right|\left|\hat{f}_{\infty}^{c}(k)\right| d n d k \\
\leq & \int_{B_{R}} \int_{S^{2}}\left|\hat{f}_{\infty}\left(k_{+}\right)\right|\left|\hat{f}_{\infty}^{c}(k)\right| d n d k \\
\leq & {\left[\int_{B_{R}} \int_{S^{2}}\left|\hat{f}_{\infty}\left(k_{+}\right)\right|^{2} d n d k\right]^{\frac{1}{2}}\left[4 \pi \int_{B_{R}}\left|\hat{f}_{\infty}(k)\right|^{2} d k\right]^{\frac{1}{2}} . }
\end{aligned}
$$

Now, we can change variables from $k$ to $k_{+}$. The equality

$$
\int_{B_{R}} \int_{S^{2}}\left|\hat{f}_{\infty}\left(k_{+}\right)\right|^{2} d n d k=\int_{B_{R}} \int_{S^{2}}\left|\hat{f}_{\infty}\left(k_{+}\right)\right|^{2} \frac{d k}{d k_{+}} d n d k_{+},
$$

thanks to the formula

$$
\frac{d k_{+}}{d k}=\left(\frac{3-e}{4}\right)^{2}\left(\frac{3-e}{4}+\frac{1+e}{4} \frac{k \cdot n}{|k|}\right) \geq\left(\frac{3-e}{4}\right)^{2}\left(\frac{1-e}{2}\right),
$$

implies

$$
\int_{B_{R}} \int_{S^{2}}\left|\hat{f}_{\infty}\left(k_{+}\right)\right|^{2} d n d k \leq \frac{32}{(3-e)^{2}(1-e)} 4 \pi \int_{B_{R}}\left|\hat{f}_{\infty}(k)\right|^{2} d k .
$$

Collecting previous estimates, we finally obtain

$$
\int_{B_{R}}\left(1+\frac{1-e^{2}}{8} \theta_{\infty}|k|^{2}\right)\left|\hat{f}_{\infty}(k)\right|^{2} d k \leq\left(\frac{32}{(3-e)^{2}(1-e)}\right)^{\frac{1}{2}} \int_{B_{R}}\left|\hat{f}_{\infty}(k)\right|^{2} d k,
$$

for any $R>0$, that we may write as

$$
\int_{B_{R}}\left|\hat{f}_{\infty}(k)\right|^{2} d k \leq \int_{B_{R}}\left(A_{1}-A_{2}|k|^{2}\right)\left|\hat{f}_{\infty}(k)\right|^{2} d k
$$

with

$$
A_{1}=\left(\frac{32}{(3-e)^{2}(1-e)}\right)^{\frac{1}{2}} \quad \text { and } \quad A_{2}=\frac{1-e^{2}}{8} \theta_{\infty} .
$$

Since the function $\left(A_{1}-A_{2}|k|^{2}\right)\left|\hat{f}_{\infty}(k)\right|^{2}$ is non-negative only in the ball of radius $R_{o}=\left(A_{1} / A_{2}\right)^{1 / 2}$, we have

$$
\int_{B_{R}}\left|\hat{f}_{\infty}(k)\right|^{2} d k \leq \int_{B_{R_{o}}}\left(A_{1}-A_{2}|k|^{2}\right)\left|\hat{f}_{\infty}(k)\right|^{2} d k \leq A_{1} \int_{B_{R_{o}}}\left|\hat{f}_{\infty}(k)\right|^{2} d k .
$$


Letting $R \rightarrow \infty$ proves that $f_{\infty} \in L^{2}\left(\mathbb{R}^{3}\right)$.

Let us consider (3.12) again. It implies

$$
\frac{1-e^{2}}{8} \theta_{\infty} \int_{B_{R}}|k|^{2}\left|\hat{f}_{\infty}(k)\right|^{2} d k \leq\left(\frac{32}{(3-e)^{2}(1-e)}\right)^{\frac{1}{2}} \int_{B_{R}}\left|\hat{f}_{\infty}(k)\right|^{2} d k,
$$

for any $R>0$, and thus $f_{\infty} \in H^{1}\left(\mathbb{R}^{3}\right)$.

Higher order regularity follows by a recursive argument. Assuming $f_{\infty} \in$ $H^{m-1}\left(\mathbb{R}^{3}\right), m \geq 2$, let us prove that $f_{\infty} \in H^{m}\left(\mathbb{R}^{3}\right)$. To this aim, multiply (3.11) by $|k|^{2(m-1)} \hat{f}_{\infty}^{c}$ and integrate on $B_{R}$. One gets

$$
\begin{aligned}
& 4 \pi \frac{1-e^{2}}{8} \theta_{\infty} \int_{B_{R}}|k|^{2 m}\left|\hat{f}_{\infty}(k)\right|^{2} d k \leq \\
& \quad \leq \int_{B_{R}} \int_{S^{2}}|k|^{2(m-1)}\left|\hat{f}_{\infty}\left(k_{-}\right) \hat{f}_{\infty}\left(k_{+}\right) \hat{f}_{\infty}^{c}(k)\right| d n d k .
\end{aligned}
$$

Proceeding as before and taking into account that $\left|k_{+}\right| \geq \frac{1-e}{2}|k|$, one shows that there exists a constant $C(m, e)$ such that

$$
\begin{aligned}
& \int_{B_{R}} \int_{S^{2}}|k|^{2(m-1)}\left|\hat{f}_{\infty}\left(k_{-}\right) \hat{f}_{\infty}\left(k_{+}\right) \hat{f}_{\infty}^{c}(k)\right| d n d k \\
& \leq C(m, e) \int_{B_{R}}|k|^{2(m-1)}\left|\hat{f}_{\infty}(k)\right|^{2} d k
\end{aligned}
$$

for any $R>0$. By induction, the right-hand side is uniformly bounded in $R>0$. Collecting previous estimates, one proves as before that there exists a constant $C\left(m, e, \theta_{\infty}\right)$ such that

$$
\int_{B_{R}}|k|^{2 m}\left|\hat{f}_{\infty}(k)\right|^{2} d k \leq C\left(m, e, \theta_{\infty}\right) \int_{B_{R}}|k|^{2(m-1)}\left|\hat{f}_{\infty}(k)\right|^{2} d k,
$$

for any $R>0$, proving the assertion. $\square$

\section{Exponential decay of the $\boldsymbol{d}_{2}$ Distance. General case}

In the previous section, we proved exponential decay of the $d_{2}$ distance of any two solutions corresponding to initial data with the same temperature. Since the temperature $\theta_{\infty}$ of the steady state is a constant which does not depend on the temperature of the initial datum, this result only shows that there is exponential decay towards the steady state for initial values that have the same temperature of the steady state. Thus, Proposition 3.1 does not answer to the natural question of the decay towards the steady state of any solution, whatever the initial temperature could be. Here, we show 
that this question can be answered positively. Indeed, the result of Proposition 3.1 can be extended to estimate explicitly the rate of convergence towards the unique equilibrium $f_{\infty}$ in terms of $d_{2}$ for general initial data without the restriction on the second moment. First, we reckon the time decay of the ratio $\theta(t) / \theta_{\infty}$.

Lemma 4.1. [Equilibration of temperature] Let $z(t)$ denote the ratio $\theta(t) / \theta_{\infty}$. Then, for any constant $\gamma>0$ it holds

$$
z^{\gamma}(t)-1 \leq \mathrm{c}(\gamma) \exp \left\{-\left(\frac{3}{2}-p\right) 2 F \theta_{\infty}^{p-1} t\right\}
$$

if $z_{0}>1$, while

$$
1-z^{\gamma}(t) \leq \mathrm{c}(\gamma) \exp \left\{-\left(\frac{3}{2}-p\right) 2 F \theta_{\infty}^{p-1} z_{0}^{\frac{1}{2}} t\right\},
$$

if $z_{0}<1$. The constant $\mathrm{c}=\mathrm{c}(\gamma)$ is given by

$$
\mathrm{c}(\gamma)=\frac{2 \gamma}{3-2 p} \max \left\{1, z_{0}^{\gamma+p-\frac{3}{2}}\right\}\left|z_{0}^{\frac{3}{2}-p}-1\right| .
$$

Proof.- From equation (2.1) it follows

$$
\begin{aligned}
\frac{d}{d t}\left(\frac{\theta}{\theta_{\infty}}\right) & =-\frac{1-e^{2}}{4} B \theta_{\infty}^{\frac{1}{2}}\left(\frac{\theta}{\theta_{\infty}}\right)^{\frac{3}{2}}+2 F \theta_{\infty}^{p-1}\left(\frac{\theta}{\theta_{\infty}}\right)^{p} \\
& =2 F \theta_{\infty}^{p-1}\left[-\left(\frac{\theta}{\theta_{\infty}}\right)^{\frac{3}{2}}+\left(\frac{\theta}{\theta_{\infty}}\right)^{p}\right] .
\end{aligned}
$$

Hence $z(t)$ satisfies the equation

$$
\frac{d z}{d t}=-2 F \theta_{\infty}^{p-1} z^{p}\left[z^{\frac{3}{2}-p}-1\right]
$$

from which we obtain easily

$$
\frac{d}{d t}\left[z^{\frac{3}{2}-p}-1\right]=-\left(\frac{3}{2}-p\right) 2 F \theta_{\infty}^{p-1} z^{\frac{1}{2}}\left[z^{\frac{3}{2}-p}-1\right] .
$$

Case $z_{0}>1$

Since $\frac{3}{2}-p>0$, equation (4.4) shows that if $z_{0}>1, z(t)$ is non-increasing and moreover $z(t) \geq 1$ for all times. Consequently (4.5) implies the inequality

$$
\frac{d}{d t}\left[z^{\frac{3}{2}-p}-1\right] \leq-\left(\frac{3}{2}-p\right) 2 F \theta_{\infty}^{p-1}\left[z^{\frac{3}{2}-p}-1\right]
$$


which after integration gives

$$
z^{\frac{3}{2}-p}-1 \leq\left(z_{0}^{\frac{3}{2}-p}-1\right) \exp \left\{-\left(\frac{3}{2}-p\right) 2 F \theta_{\infty}^{p-1} t\right\} .
$$

Hence

$$
z(t) \leq\left\{1+\left(z_{0}^{\frac{3}{2}-p}-1\right) \exp \left\{-\left(\frac{3}{2}-p\right) 2 F \theta_{\infty}^{p-1} t\right\}\right\}^{\frac{2}{3-2 p}},
$$

and, for any power $\gamma>0$

$$
z^{\gamma}(t)-1 \leq\left\{1+\left(z_{0}^{\frac{3}{2}-p}-1\right) \exp \left\{-\left(\frac{3}{2}-p\right) 2 F \theta_{\infty}^{p-1} t\right\}\right\}^{\frac{2 \gamma}{3-2 p}}-1
$$

The right hand side of this last inequality is a function of the form

$$
y(x)=(1+a x)^{q}-1
$$

where

$$
q=\frac{2 \gamma}{3-2 p}, \quad a=z_{0}^{\frac{3}{2}-p}-1, \quad x=\exp \left\{-\left(\frac{3}{2}-p\right) 2 F \theta_{\infty}^{p-1} t\right\} .
$$

By Taylor formula $y(x)=q(1+a \bar{x})^{q-1} a x$; since in our case $0 \leq \bar{x} \leq 1$ it holds

$$
\begin{array}{ll}
(1+a \bar{x})^{q-1} \leq 1 & \text { if } q-1<0, \\
(1+a \bar{x})^{q-1} \leq(1+a)^{q-1} & \text { if } q-1>0 .
\end{array}
$$

Thus

$$
y(x) \leq q \max \left\{1,(1+a)^{q-1}\right\} a x .
$$

By inserting expressions (4.7) into (4.8), we find inequality (4.1) which shows the exponential decay of $z^{\gamma}(t)-1$ for all values of $\gamma>0$.

Case $z_{0}<1$.

The proof is very similar to the previous one. If $z_{0}<1$, equation (4.4) implies that $z(t)$ is non-decreasing, so that $z(t) \geq z_{0}$ for all times. Thus, equation (4.5) implies

$$
\frac{d}{d t}\left[1-z^{\frac{3}{2}-p}\right] \leq-\left(\frac{3}{2}-p\right) 2 F \theta_{\infty}^{p-1} z_{0}^{\frac{1}{2}}\left[1-z^{\frac{3}{2}-p}\right],
$$

from which it follows

$$
1-z^{\frac{3}{2}-p} \leq\left(1-z_{0}^{\frac{3}{2}-p}\right) \exp \left\{-\left(\frac{3}{2}-p\right) 2 F \theta_{\infty}^{p-1} z_{0}^{\frac{1}{2}} t\right\} .
$$


Hence

$$
z(t) \geq\left\{1-\left(1-z_{0}^{\frac{3}{2}-p}\right) \exp \left\{-\left(\frac{3}{2}-p\right) 2 F \theta_{\infty}^{p-1} z_{0}^{\frac{1}{2}} t\right\}\right\}^{\frac{2}{3-2 p}}
$$

from which it follows

$$
1-z^{\gamma}(t) \leq 1-\left\{1-\left(1-z_{0}^{\frac{3}{2}-p}\right) \exp \left\{-\left(\frac{3}{2}-p\right) 2 F \theta_{\infty}^{p-1} z_{0}^{\frac{1}{2}} t\right\}\right\}^{\frac{2 \gamma}{3-2 p}}
$$

The function on the right hand side is of the form

$$
y(x)=1-(1-a x)^{q},
$$

with

$$
q=\frac{2 \gamma}{3-2 p}, \quad a=1-z_{0}^{\frac{3}{2}-p}, \quad x=\exp \left\{-\left(\frac{3}{2}-p\right) 2 F \theta_{\infty}^{p-1} z_{0}^{\frac{1}{2}} t\right\} .
$$

Taylor formula gives $y(x)=q(1-a \bar{x})^{q-1} a x$. Since $0 \leq \bar{x} \leq 1$,

$$
\begin{array}{ll}
(1-a \bar{x})^{q-1} \leq(1-a)^{q-1} & \text { if } q-1<0, \\
(1-a \bar{x})^{q-1} \leq 1 & \text { if } q-1>0 .
\end{array}
$$

Finally

$$
y(x) \leq q \max \left\{1,(1-a)^{q-1}\right\} a x,
$$

and by inserting expressions (4.10) into (4.11) we find inequality (4.2). We remark that in this second case the decay is slower, and it depends on $z_{0}<1$. $\square$

Theorem 4.2. [Exponential decay of the $\boldsymbol{d}_{2}$ distance] Any solution $f(t, v)$ of (1.1) corresponding to an initial density with unit mass, zero mean velocity and finite initial temperature, converges exponentially towards the steady state $f_{\infty}(v)$ in $d_{2}$ distance. More precisely, there exist constants $C_{1}, C_{2}, C_{3}>0$ such that

$$
d_{2}\left(\hat{f}(t), \hat{f}_{\infty}\right) \leq d_{2}\left(\hat{f}_{0}, \hat{f}_{\infty}\right) \mathrm{e}^{-\left(1-\gamma_{0}\right) \mathrm{C}_{1} \mathrm{t}}+\mathrm{C}_{2} \mathrm{e}^{-\mathrm{C}_{3} \mathrm{t}}
$$

for all $t \geq 0$, with $\gamma_{0}=\left(e^{2}+3\right) / 4<1$.

Proof.- We proceed exactly as in the proof of Proposition 3.1. Therefore we will only sketch the proof, and put the emphasis on the differences. In agreement with the notations introduced in Lemma 4.1, the variable $\theta(t)$ will be replaced by $\theta_{\infty} z(t)$. Since the temperatures of the two solutions are different, with respect to the computations of Proposition 3.1, we have 
an additional term that we may write using the equation (3.10) satisfied by $f_{\infty}$. We have

$$
\begin{aligned}
& \frac{\partial}{\partial t} \frac{\hat{f}(k)-\hat{f}_{\infty}(k)}{|k|^{2}}= \frac{B}{4 \pi} \theta_{\infty}^{\frac{1}{2}} z^{\frac{1}{2}}(t) \int_{S^{2}}\left[\frac{\hat{f}\left(k_{-}\right) \hat{f}\left(k_{+}\right)-\hat{f}_{\infty}\left(k_{-}\right) \hat{f}_{\infty}\left(k_{+}\right)}{|k|^{2}}\right. \\
&\left.-\frac{\hat{f}(0) \hat{f}(k)-\hat{f}_{\infty}(0) \hat{f}_{\infty}(k)}{|k|^{2}}\right] d n \\
& \\
&-F \theta_{\infty}^{p} z^{p}(t)|k|^{2} \frac{\hat{f}(k)-\hat{f}_{\infty}(k)}{|k|^{2}} \\
& \\
&+F \theta_{\infty}^{p}\left(z^{\frac{1}{2}}(t)-z^{p}(t)\right) \hat{f}_{\infty}(k) .
\end{aligned}
$$

Equation (3.6) becomes in our case

$$
\begin{aligned}
\left|\partial_{t} h(t, k)+\left(B \theta_{\infty}^{\frac{1}{2}} z^{\frac{1}{2}}(t)+F \theta_{\infty}^{p} z^{p}(t)|k|^{2}\right) h(t, k)\right| \\
\leq \gamma_{0} B \theta_{\infty}^{\frac{1}{2}} z^{\frac{1}{2}}(t)\|h(t, \cdot)\|_{\infty}+\varphi(t)
\end{aligned}
$$

where

$$
h(t, k)=\frac{\hat{f}(k)-\hat{f}_{\infty}(k)}{|k|^{2}},
$$

and

$$
\varphi(t)=F \theta_{\infty}^{p}\left|z^{\frac{1}{2}}(t)-z^{p}(t)\right| .
$$

Proceeding as in Proposition 3.1, we obtain

$$
\begin{aligned}
\exp \left(B \theta_{\infty}^{\frac{1}{2}} \int_{0}^{t} z^{\frac{1}{2}}(s) d s\right)\|h(t, \cdot)\|_{\infty} \leq\|h(0, \cdot)\|_{\infty}+\Phi(t) \\
\quad+\gamma_{0} B \theta_{\infty}^{\frac{1}{2}} \int_{0}^{t} z^{\frac{1}{2}}(\tau)\|h(\tau, \cdot)\|_{\infty} \exp \left(B \theta_{\infty}^{\frac{1}{2}} \int_{0}^{\tau} z^{\frac{1}{2}}(s) d s\right) d \tau
\end{aligned}
$$

where

$$
\Phi(t)=\int_{0}^{t} \varphi(\tau) \exp \left(B \theta_{\infty}^{\frac{1}{2}} \int_{0}^{\tau} z^{\frac{1}{2}}(s) d s\right) d \tau .
$$


By the generalized Gronwall lemma [21], denoting by $w(t)$ the same quantity as in Proposition 3.1, we finally conclude that

$$
\begin{aligned}
w(t) & \leq w(0) \exp \left(\gamma_{0} B \theta_{\infty}^{\frac{1}{2}} \int_{0}^{t} z^{\frac{1}{2}}(\tau) d \tau\right) \\
& +\int_{0}^{t} \exp \left(\gamma_{0} B \theta_{\infty}^{\frac{1}{2}} \int_{\tau}^{t} z^{\frac{1}{2}}(s) d s\right) \varphi(\tau) \exp \left(B \theta_{\infty}^{\frac{1}{2}} \int_{0}^{\tau} z^{\frac{1}{2}}(s) d s\right) d \tau .
\end{aligned}
$$

Hence

$$
\|h(t, \cdot)\|_{\infty} \leq\|h(0, \cdot)\|_{\infty} \exp \left(-\left(1-\gamma_{0}\right) B \theta_{\infty}^{\frac{1}{2}} \int_{0}^{t} z^{\frac{1}{2}}(s) d s\right)+\Psi(t)
$$

where

$$
\begin{aligned}
\Psi(t)=\exp \left(-\left(1-\gamma_{0}\right) B\right. & \left.\theta_{\infty}^{\frac{1}{2}} \int_{0}^{t} z^{\frac{1}{2}}(s) d s\right) \int_{0}^{t} \varphi(\tau) \\
& \times \exp \left(\left(1-\gamma_{0}\right) B \theta_{\infty}^{\frac{1}{2}} \int_{0}^{\tau} z^{\frac{1}{2}}(s) d s\right) d \tau .
\end{aligned}
$$

To finish the proof, it suffices to show that $\Psi(t)$ vanishes for $t \rightarrow \infty$. First of all, notice that definition (4.15) implies that if $p=\frac{1}{2}$ the function $\varphi(\tau)$ vanishes (thus no further proof is needed), while for $p \neq \frac{1}{2}$

$$
\varphi(\tau) \leq F \theta_{\infty}^{p}\left(\left|z^{\frac{1}{2}}(\tau)-1\right|+\left|z^{p}(\tau)-1\right|\right) .
$$

Therefore $\varphi(\tau)$ can be estimated using inequalities (4.1)-(4.2). 
Case $z_{0}>1$.

The function $\Psi(t)$ can be bounded in the following way:

$$
\begin{aligned}
& \Psi(t)=\exp \left(-\left(1-\gamma_{0}\right) B \theta_{\infty}^{\frac{1}{2}} \int_{0}^{t}\left[z^{\frac{1}{2}}(s)-1\right] d s-\left(1-\gamma_{0}\right) B \theta_{\infty}^{\frac{1}{2}} t\right) \\
& \quad \times \int_{0}^{t} \varphi(\tau) \exp \left(\left(1-\gamma_{0}\right) B \theta_{\infty}^{\frac{1}{2}} \int_{0}^{\tau}\left[z^{\frac{1}{2}}(s)-1\right] d s+\left(1-\gamma_{0}\right) B \theta_{\infty}^{\frac{1}{2}} \tau\right) d \tau \\
& \leq \exp \left(-\left(1-\gamma_{0}\right) B \theta_{\infty}^{\frac{1}{2}} t\right) \int_{0}^{t} \varphi(\tau) \exp \left(\left(1-\gamma_{0}\right) B \theta_{\infty}^{\frac{1}{2}} \tau\right) d \tau
\end{aligned}
$$

We used the bounds $z^{\frac{1}{2}}(s)-1>0$ and $\tau<t$. Now, taking into account formula (4.1),

$$
\begin{aligned}
& \Psi(t) \leq \\
& \leq \mathcal{C} \exp \left(-\left(1-\gamma_{0}\right) B \theta_{\infty}^{\frac{1}{2}} t\right) \int_{0}^{t} \exp \left\{\left[-\left(\frac{3}{2}-p\right) 2 F \theta_{\infty}^{p-1}+\left(1-\gamma_{0}\right) B \theta_{\infty}^{\frac{1}{2}}\right] \tau\right\} d \tau \\
& =\mathcal{C} \frac{\exp \left(-\left(\frac{3}{2}-p\right) 2 F \theta_{\infty}^{p-1} t\right)-\exp \left(-\left(1-\gamma_{0}\right) B \theta_{\infty}^{\frac{1}{2}} t\right)}{-\left(\frac{3}{2}-p\right) 2 F \theta_{\infty}^{p-1}+\left(1-\gamma_{0}\right) B \theta_{\infty}^{\frac{1}{2}}}
\end{aligned}
$$

where

$$
\begin{aligned}
\mathcal{C} & =F \theta_{\infty}^{p}\left[\mathrm{c}\left(\frac{1}{2}\right)+\mathrm{c}(p)\right] \\
& =\frac{F \theta_{\infty}^{p}}{3-2 p}\left\{\max \left\{1, z_{0}^{p-1}\right\}+2 p \max \left\{1, z_{0}^{2 p-\frac{3}{2}}\right\}\right\}\left|z_{0}^{\frac{3}{2}-p}-1\right| .
\end{aligned}
$$

Hence $\Psi(t)$ decays exponentially and the exponential decay of $d_{2}$ is proved. Depending on the sign of

$$
-\left(\frac{3}{2}-p\right) 2 F \theta_{\infty}^{p-1}+\left(1-\gamma_{0}\right) B \theta_{\infty}^{\frac{1}{2}}
$$

(which vanishes for $p=\frac{1}{2}$ ), we conclude that

$$
C_{3}=\left\{\begin{array}{ll}
\left(\frac{3}{2}-p\right) 2 F \theta_{\infty}^{p-1} & , \quad \text { if } p>\frac{1}{2} \\
\left(1-\gamma_{0}\right) B \theta_{\infty}^{\frac{1}{2}} & , \quad \text { if } p<\frac{1}{2}
\end{array} .\right.
$$

Case $z_{0}<1$. 
The proof is a bit more complicated than the previous one, because now $z^{\frac{1}{2}}(s)-1<0$. To estimate $\Psi(t)$, using (4.2) we recover the bound

$$
\begin{aligned}
& \exp \left(\left(1-\gamma_{0}\right) B \theta_{\infty}^{\frac{1}{2}} \int_{0}^{t}\left[1-z^{\frac{1}{2}}(s)\right] d s\right) \\
\leq & \exp \left\{\left(1-\gamma_{0}\right) \frac{1}{(3-2 p)^{2}} \frac{B}{F} z_{0}^{-\frac{1}{2}} \theta_{\infty}^{\frac{3}{2}-p} \max \left\{1, z_{0}^{p-1}\right\}\left(1-z_{0}^{\frac{3}{2}-p}\right)\right\}=: \mathcal{K} .
\end{aligned}
$$

Precisely we have

$$
\begin{aligned}
& \Psi(t)=\exp \left(\left(1-\gamma_{0}\right) B \theta_{\infty}^{\frac{1}{2}} \int_{0}^{t}\left[1-z^{\frac{1}{2}}(s)\right] d s-\left(1-\gamma_{0}\right) B \theta_{\infty}^{\frac{1}{2}} t\right) \\
& \quad \times \int_{0}^{t} \varphi(\tau) \exp \left(-\left(1-\gamma_{0}\right) B \theta_{\infty}^{\frac{1}{2}} \int_{0}^{\tau}\left[1-z^{\frac{1}{2}}(s)\right] d s+\left(1-\gamma_{0}\right) B \theta_{\infty}^{\frac{1}{2}} \tau\right) d \tau \\
& \leq \mathcal{K} \exp \left(-\left(1-\gamma_{0}\right) B \theta_{\infty}^{\frac{1}{2}} t\right) \int_{0}^{t} \varphi(\tau) \exp \left(\left(1-\gamma_{0}\right) B \theta_{\infty}^{\frac{1}{2}} \tau\right) d \tau .
\end{aligned}
$$

Applying again formula (4.2) to the function $\varphi(\tau)$, it follows

$$
\begin{aligned}
\Psi(t) \leq & \mathcal{C K} \exp \left(-\left(1-\gamma_{0}\right) B \theta_{\infty}^{\frac{1}{2}} t\right) \\
& \times \int_{0}^{t} \exp \left\{\left[-\left(\frac{3}{2}-p\right) 2 F \theta_{\infty}^{p-1} z_{0}^{\frac{1}{2}}+\left(1-\gamma_{0}\right) B \theta_{\infty}^{\frac{1}{2}}\right] \tau\right\} d \tau \\
= & \mathcal{C} \mathcal{K} \frac{\exp \left(-\left(\frac{3}{2}-p\right) 2 F \theta_{\infty}^{p-1} z_{0}^{\frac{1}{2}} t\right)-\exp \left(-\left(1-\gamma_{0}\right) B \theta_{\infty}^{\frac{1}{2}} t\right)}{-\left(\frac{3}{2}-p\right) 2 F \theta_{\infty}^{p-1} z_{0}^{\frac{1}{2}}+\left(1-\gamma_{0}\right) B \theta_{\infty}^{\frac{1}{2}}}
\end{aligned}
$$

where $\mathcal{C}$ is the same constant obtained in the previous case. Also in this case $\Psi(t)$ decreases at an exponential rate. Let us remark again that the previous decay holds if

$$
-\left(\frac{3}{2}-p\right) 2 F \theta_{\infty}^{p-1} z_{0}^{\frac{1}{2}}+\left(1-\gamma_{0}\right) B \theta_{\infty}^{\frac{1}{2}}
$$

does not vanish. Depending on the sign of this factor then one obtains the value of $C_{3}$ similarly to the case $z_{0}>1$. 
Remark 4.3. Theorems 3.2-4.2 can be stated also for the inelastic onedimensional Kac model introduced in Ref. [19]:

$$
\frac{\partial \hat{f}}{\partial t}=\frac{\sqrt{\theta(t)}}{2 \pi} \int_{0}^{2 \pi}\left[\hat{f}\left(k_{p}^{-}\right) \hat{f}\left(k_{p}^{+}\right)-\hat{f}(k) \hat{f}(0)\right] d \vartheta-F \theta^{p}(t)|k|^{2} \hat{f}
$$

where

$$
k_{p}^{-}=k \sin \vartheta|\sin \vartheta|^{p} \quad k_{p}^{+}=k \cos \vartheta|\cos \vartheta|^{p} .
$$

Remark 4.4. The theorems proved in this section imply the convergence of other probability metrics that turn out to be equivalent to $d_{s}$, that is metrics which define the same weak $k^{\star}$-uniformity on the class of probability distributions having bounded the moment of order $s[15,21]$.

\section{Propagation of Smoothness for the Boltzmann equation}

The goal of this section is to show that the smoothness of the initial data of the Boltzmann equation is propagated so that we have bounds on the smoothness uniform in time. Using the same computations of step 3 of Theorem 3.2 , we obtain the inequality

$$
\begin{aligned}
& \frac{d}{d t} \int_{\mathbb{R}^{3}}|k|^{2 m}|\hat{f}(k)|^{2} d k \leq \\
& 2 B \sqrt{\theta(t)}\left[\frac{1}{4 \pi} \int_{S^{2}} \int_{\mathbb{R}^{3}}|k|^{2 m}\left|\hat{f}\left(k_{-}\right) \hat{f}\left(k_{+}\right) \hat{f}^{c}(k)\right| d n d k-\int_{\mathbb{R}^{3}}|k|^{2 m}|\hat{f}(k)|^{2} d k\right] \\
& \quad-2 F \theta(t)^{p} \int_{\mathbb{R}^{3}}|k|^{2 m+2}|\hat{f}(k)|^{2} d k .
\end{aligned}
$$

Let us remark that, thanks to the bound (3.13)

$$
\int_{\mathbb{R}^{3}} \frac{1}{4 \pi} \int_{S^{2}}|k|^{2 m}\left|\hat{f}\left(k_{-}\right) \hat{f}\left(k_{+}\right) \hat{f}^{c}(k)\right| d n d k \leq C(m, e) \int_{\mathbb{R}^{3}}|k|^{2 m}|\hat{f}(k)|^{2} d k,
$$

where $C(m, e)>1$ is a constant which depends only on $m$ and on the coefficient of restitution $e$, and can be explicitly computable. Hence, if $Z_{m}(t)=\|f(t)\|_{H^{m}}, Z_{m}(t)$ satisfies the inequality

$$
\frac{d Z_{m}(t)}{d t} \leq 2 B \sqrt{\theta(t)}[C(m, e)-1] Z_{m}(t)-2 F \theta(t)^{p} Z_{m+1}(t) .
$$

The desired result follows from (5.2) by virtue of the following Nash-type inequality.

Lemma 5.1. Let $f \in H^{m+1}\left(\mathbb{R}^{3}\right)$ be a probability density function. Then, $f \in H^{m}\left(\mathbb{R}^{3}\right)$ and the following bound holds

$$
\|f\|_{H^{m+1}\left(\mathbb{R}^{3}\right)} \geq c_{m}\left(\|f\|_{H^{m}\left(\mathbb{R}^{3}\right)}\right)^{(2 m+5) /(2 m+3)}
$$


where

$$
c_{m}=\left(\frac{1}{2 \pi}\right)^{2 /(2 m+3)}\left(\frac{2 m+3}{2 m+5}\right)^{(2 m+5) /(2 m+3)} .
$$

Proof.- For any constant $R>0$, we obtain the bound

$\int_{\mathbb{R}^{3}}|k|^{2 m}|\hat{f}(k)|^{2} d k \leq \int_{|k| \leq R}|k|^{2 m}|\hat{f}(k)|^{2} d k+\frac{1}{R^{2}} \int_{|k|>R}|k|^{2 m+2}|\hat{f}(k)|^{2} d k$.

Since $f$ is a probability density, $|\hat{f}(k)| \leq 1$. Hence

$$
\int_{|k| \leq R}|k|^{2 m}|\hat{f}(k)|^{2} d k \leq \int_{|k| \leq R}|k|^{2 m} d k=4 \pi \frac{R^{2 m+3}}{2 m+3} .
$$

By hypothesis, $f$ belongs to $H^{m+1}\left(\mathbb{R}^{3}\right)$. This implies the inequality

$$
\|f\|_{H^{m}\left(\mathbb{R}^{3}\right)} \leq 4 \pi \frac{R^{2 m+3}}{2 m+3}+\frac{1}{R^{2}}\|f\|_{H^{m+1}\left(\mathbb{R}^{3}\right)} .
$$

Optimizing in $R$ now yields the result.

We use inequality (5.3) into (5.2) to obtain

$$
\frac{d Z_{m}(t)}{d t} \leq 2 B \sqrt{\theta(t)}[C(m, e)-1] Z_{m}(t)-2 F \theta(t)^{p} c_{m}\left(Z_{m}(t)\right)^{(2 m+5) /(2 m+3)} .
$$

Inequality (5.5) can we written as

$$
\begin{aligned}
& \frac{d Z_{m}(t)}{d t} \leq \\
& \quad \leq 2 B \sqrt{\theta(t)}[C(m, e)-1] Z_{m}(t)\left\{1-\frac{F \theta(t)^{p-1 / 2} c_{m}}{B[C(m, e)-1]}\left(Z_{m}(t)\right)^{2 /(2 m+3)}\right\},
\end{aligned}
$$

that gives the bound

$$
Z_{m}(t) \leq \max \left\{Z_{m}(0) ;\left[\frac{B[C(m, e)-1]}{F \theta(t)^{p-1 / 2} c_{m}}\right]^{(2 m+3) / 2}\right\}
$$

Considering that the temperature $\theta(t)$ is bounded uniformly in time both from below and above, we conclude that $Z_{m}(t)$ is uniformly bounded in time.

Theorem 5.2. [Propagation of smoothness] Let $f_{0}$ be any initial datum for equation (1.1) with $\left\|f_{0}\right\|_{H^{m}\left(\mathbb{R}^{3}\right)}$ finite. Then, any probability density solution $f(t, v)$ of $(1.1)$ is bounded in $H^{m}\left(\mathbb{R}^{3}\right)$, and there is a universal constant $C_{m}$ so that, for all $t>0$,

$$
\|f(t)\|_{H^{m}\left(\mathbb{R}^{3}\right)} \leq \max \left\{\left\|f_{0}\right\|_{H^{m}\left(\mathbb{R}^{3}\right)}, C_{m}\right\} .
$$




\section{Strong Convergence to Equilibrium}

This section is very short. To prove Theorem 1.3, we only need to collect results and to explain how to compute the constants involved in it. The key point are some interpolation inequalities, recently considered in [9] to obtain a result similar to Theorem 1.3 but for the elastic Boltzmann equation for Maxwell molecules. The first of these inequalities reads

Lemma 6.1. [Control of the $\boldsymbol{H}^{\boldsymbol{m}}$-distance [9, Theorem 4.1]] Let $m \geq 0$, and $\beta_{1}, \beta_{2}>0,0<\beta_{2}<1$ be given. Then

$$
\|f-g\|_{H^{m}\left(\mathbb{R}^{n}\right)} \leq C\left(\beta_{1}, \beta_{2}\right) d_{2}(f, g)^{2\left(1-\beta_{2}\right)}\left(\|f\|_{H^{r}\left(\mathbb{R}^{N}\right)}+\|g\|_{H^{r}\left(\mathbb{R}^{N}\right)}\right),
$$

with

$$
\begin{gathered}
r=\frac{2 m+\left(8+\beta_{1}+N\right)\left(1-\beta_{2}\right)}{2 \beta_{2}}, \\
C\left(\beta_{1}, \beta_{2}\right)=\left(\left|B^{N}\right|\left(1+N / \beta_{1}\right)\right)^{1-\beta_{2}},
\end{gathered}
$$

and where $\left|B^{N}\right|$ denotes the volume of the unit ball in $\mathbb{R}^{N}$.

This result shows that the weak $d_{2}$ distance coupled with $\|\cdot\|_{H^{r}}$ smoothness, controls the $H^{m}$ distance for $r$ sufficiently larger than $m$. The next inequality shows that control of the sufficiently many moments and control of the $L^{2}$ norm together, control the $L^{1}$ norm.

Lemma 6.2. [Control of the $\boldsymbol{L}^{\mathbf{1}}$-distance [9, Theorem 4.2]] Let $f$ be an integrable function on $\mathbb{R}^{N}$. Then, for all $r>0$

$$
\begin{aligned}
& \int_{\mathbb{R}^{N}}|f(v)| d v \leq \\
& \quad \leq C(N, r)\left(\int_{\mathbb{R}^{N}}|f(v)|^{2} d v\right)^{2 r /(N+4 r)}\left(\int_{\mathbb{R}^{N}}|v|^{2 r}|f(v)| d v\right)^{N /(N+4 r)}
\end{aligned}
$$

with

$$
C(N, r)=\left[\left(\frac{N}{4 r}\right)^{4 r /(N+4 r)}+\left(\frac{4 r}{N}\right)^{N /(N+4 r)}\right]\left|B^{N}\right|^{2 r /(N+4 r)}
$$

Proof of Theorem 1.3. Since we have established Lemma 2.3 in Section 2, Theorem 5.2 in Section 5, Theorem 1.3 follows from the interpolation inequalities, Lemmas 6.1 and 6.2 above.

Acknowledgements: The authors acknowledge support from the European IHP network "Hyperbolic and Kinetic Equations: Asymptotics, Numerics, Applications" HPRN-CT-2002-00282. JAC acknowledges the support from DGI-MCYT/FEDER project BFM2002-01710 and CIRIT 
2001SGR/00172. GT acknowledges support from the Italian MIUR project "Mathematical Problems of Kinetic Theories".

\section{REFERENCES}

[1] D. Benedetto, E. Caglioti, J.A. Carrillo, M. Pulvirenti, "A non-maxwellian steady distribution for one-dimensional granular media", J. Stat. Phys. 91 (1998), 979-990.

[2] C. Bizon, M.D. Shattuck, J.B. Swift, H.L. Swinney, "Transport coefficients for granular media from molecular dynamics simulations", Phys. Rev. E 60 (1999), 43404351.

[3] A.V. Bobylev, "The theory of the nonlinear spatially uniform Boltzmann equation for Maxwell molecules", Sov. Sci. Rev. C. Math. Phys. 7 (1988), 111-233.

[4] A.V. Bobylev, J.A. Carrillo, I. Gamba, "On some properties of kinetic and hydrodynamics equations for inelastic interactions", J. Statist. Phys. 98 (2000), 743-773.

[5] A.V. Bobylev, C. Cercignani, "Moment equations for a Granular Material in a Thermal Bath", J. Statist. Phys. 106 (2002), 547-567.

[6] A.V. Bobylev, C. Cercignani, "Self-similar asymptotics for the Boltzmann equation with inelastic and elastic interactions", J. Statist. Phys. 110 (2003), 333-375.

[7] A.V. Bobylev, C. Cercignani, G. Toscani, "Proof of an asymptotic property of selfsimilar solutions of the Boltzmann equation for granular materials", J. Statist. Phys. 111 (2003), 403-417.

[8] A.V. Bobylev, I. Gamba, V. Panferov, "Moment inequalities and high-energy tails for Boltzmann equations with inelastic interactions", to appear in J. Statist. Phys.

[9] E.A. Carlen, E. Gabetta, G. Toscani, "Propagation of smoothness and the rate of exponential convergence to equilibrium for a spatially homogeneous Maxwellian gas", Commun. Math. Phys. 305 (1999) 521-546.

[10] J.A. Carrillo, R.J. McCann, C. Villani, "Contractions in the 2-Wasserstein length space and thermalization of granular media", HYKE preprint (2004) (www.hyke.org).

[11] J.A. Carrillo, C. Cercignani, I. Gamba, "Steady states of a Boltzmann equation for driven granular media", Phys. Rev. E 62 (2000), 7700-7707.

[12] J.A. Carrillo, G. Toscani, "Exponential convergence toward equilibrium for homogeneous Fokker-Planck-type equations", Math. Meth. in the Appl. Sci 21 (1998), 1269-1286.

[13] J.A. Carrillo, G. Toscani, "Wasserstein metric and large-time asymptotics of nonlinear diffusion equations", HYKE preprint (2004) (www.hyke.org).

[14] C. Cercignani, R. Illner, C. Stoica, "On Diffusive Equilibria in Generalized Kinetic Theory", J. Statist. Phys. 105 (2001), 337-352.

[15] E. Gabetta, G. Toscani, W. Wennberg, "Metrics for Probability Distributions and the Trend to Equilibrium for Solutions of the Boltzmann Equation", J. Statist. Phys. 81 (1995), 901-934.

[16] I. Gamba, V. Panferov, C. Villani, "On the Boltzmann equation for diffusively excited granular media", to appear in Comm. Math. Phys.

[17] I. Gamba, V. Panferov, C. Villani, "On the maximum principles and pointwise upper estimates for Boltzmann equations for granular media", preprint.

[18] H. Li, G. Toscani, "Long-time asymptotics of kinetic models of granular flows", to appear in Arch. Rat. Mech. Anal.

[19] A. Pulvirenti, G. Toscani, "Asymptotic properties of the inelastic Kac model", J. Statist. Phys. 114 (2004), 1453-1480. 
[20] G. Toscani, "Kinetic and Hydrodynamical models of nearly elastic granular flows", to appear in Monat. Math.

[21] G. Toscani, C. Villani, "Probability Metrics and Uniqueness of the Solution to the Boltzmann Equation for a Maxwell Gas", J. Statist. Phys. 94 (1999), 619-637.

[22] D.R.M. Williams, F.C. MacKintosh, "Driven granular media in one dimension: Correlations and equation of state", Phys. Rev. E 54 (1996), R9-R12.

\section{Bisi,}

Dipartimento di Matematica "F. Enriques", Università di Milano,

via Saldini 50, 20133 Milano, Italy.

E-mail: bisi@mat.unimi.it

J. A. Carrillo,

ICREA (Institució Catalana de Recerca i Estudis Avançats) and Departament de Matemàtiques,

Universitat Autònoma de Barcelona,

E-08193 Bellaterra,

E-mail: carrillo@mat.uab.es

G. Toscani,

Dipartimento di Matematica,

Università di Pavia,

via Ferrata 1, I-27100 Pavia, Italy.

E-mail: toscani@dimat.unipv.it 